\title{
Role of 3D printing in surgical education for robotic urology procedures
}

\author{
Ahmed E. Ghazi, Brett A. Teplitz \\ University of Rochester Medical Center (URMC), Rochester, NY, USA \\ Contributions: (I) Conception and design: AE Ghazi; (II) Administrative support: BA Teplitz; (III) Provision of study materials or patients: None; \\ (IV) Collection and assembly of data: All authors; (V) Data analysis and interpretation: All authors; (VI) Manuscript writing: All authors; (VII) Final \\ approval of manuscript: All authors. \\ Correspondence to: Ahmed E. Ghazi. Associate Professor of Urology, George W. Conner Deans Teaching Fellow, Endourology Fellowship Associate \\ Program Director, Director of Simulation Innovation laboratory, University of Rochester Medical Center (URMC), 601 Elmwood Avenue, \\ Rochester, NY 14642, USA. Email: Ahmed_Ghazi@URMC.Rochester.edu.
}

\begin{abstract}
During the past 5 years, the body of literature surrounding the utilization of three-dimensional (3D) printing in the field of urology has grown exponentially. Incentivized by work hour restrictions, patient safety initiatives, and inspired by technical advances in biomaterials and rapid printing strategies, this emerging, and fascinating area of research has begun to make headway into clinical practice. However, concerns about cost, limited understanding of the technical processes involved, and lack of its potential uses remain barriers to its widespread adoption. We examined existing published literature on how 3D printing technologies have been utilized in the field of Urology to enhance pre-operative planning, revitalize surgical training, and modernize patient education, with particular focus on, robotic surgery. To date, 3D-printed models have been used and studied most commonly in the preoperative planning for nephron-sparing surgeries during the treatment of renal masses, where the challenges of complex renal anatomy and benefits of reducing renal ischemic injury create the most intuitive value. Prostate models are the second most common, particularly in the planning of nerve-sparing procedures. Early studies have demonstrated sufficient realism and educational effectiveness. Subsequent studies demonstrated improved surgeon confidence, operative performance, and optimized patient outcomes including high levels of patient satisfaction. Realistic, accurate, and reasonably priced models can currently be generated within hours using standard desktop 3D printers. While primarily utilized as anatomic replicas of diseased organs that restore a sense of haptic feedback lost in robotic procedures, innovations in polymers, improvements in $3 \mathrm{D}$ printer host and modeling software, and upgrades in printer hardware allow this technology to serve as a comprehensive, interactive, simulation platform that can be a critical surgical decision making as well as an effective teaching tool. As Urologists continue to rapidly diversify and iterate upon this adaptive modality, the benefits in patient outcomes will likely outpace the diminishing drawbacks, and we may well see the next revolution in surgical education, robotic techniques, and personalized medicine concurrently.
\end{abstract}

Keywords: Three-dimensional printing (3D printing); patient-specific simulation; robotic surgery; surgical simulation; surgical training; patient education

Submitted Oct 01, 2019. Accepted for publication Dec 16, 2019.

doi: $10.21037 /$ tau. 2020.01.03

View this article at: http://dx.doi.org/10.21037/tau.2020.01.03 


\section{Introduction}

Ever since Charles Hull first developed stereolithography in 1983, using UV light to cure and bond photopolymer resins, the world of three-dimensional (3D) printing has experienced exponential growth. The fields of orthopedics and maxillofacial surgery were among the earliest adopters $(1,2)$. Urology, has emerged as a "hotbed" of innovation in the last decade, due in part to a confluence of developments in minimally invasive surgery and a push towards simulation-based training which have fostered an environment for continued innovation in the field of robotic surgery education. Various centers around the world have utilized this technology to print individualized physical models from patients radiological imaging which aid in the processes of informed consent, enhance resident education, and refine preoperative robotic surgical planning. We must continue to innovate and strive to advance this dynamic technology if the field is to develop beyond fabricating simple visual aids, and provide an interactive platform with the authenticity necessary to conduct an operation in its entirety.

\section{Definitions/background}

$3 \mathrm{D}$ printing is defined as an additive manufacturing process that produces a $3 \mathrm{D}$ structure from a series of $2 \mathrm{D}$ images, which is distinct from traditional manufacturing that involves a progressive, subtractive process. In the medical realm, the process generally begins with a highquality image file in Digital Imaging and Communications in Medicine (DICOM) format, primarily MRI, CT or any axial imaging. These DICOM files are then converted to a computer-aided design (CAD) code, such as standard tessellation language (STL), which forms a virtual 3D object. At the core of $3 \mathrm{D}$ printing is a piece of software called the slicer, which utilizes the CAD model as a guide to generate the instructions for the printer itself in the form of a G-code bundle to build the 3D object (3). Advances in software have allowed the coding of additional data points such as color and texture into the file formats, and postprocessing tools can help refine the model to achieve highly accurate and reproducible reconstructions. Despite all these advances, the most commonly used materials for the fabrication of $3 \mathrm{D}$ printed education models in the surgical field are nonresectable resins. These resins lack the dynamic and biomechanical properties of real tissues that are necessary to be effectively utilized as a hands-on platform to practice and rehearse a surgical procedure (4).

\section{Applications in urology}

Before any new technology can be accepted and implemented broadly, it must pass the tests of feasibility and accuracy. 3D printing in the field of Urology started as a visual guide that could potentially influence the surgeons' understanding of renal malignancies (5). Several publications have also reported using $3 \mathrm{D}$ technology to develop simulation platforms for hands-on resident training or rehearsals by practicing surgeons (6). Meanwhile, numerous other studies have highlighted novel and effective uses of these resources, as surgical education tools for patients and medical students. These applications can be grouped into the following categories (Table S1):

(I) Patient-specific 3D models for guidance, planning, and rehearsal of a complex procedure to benefit practicing surgeons;

(II) Generic 3D procedural models for surgical training (hands-on surgical practice) for novice surgeons;

(III) 3D models for patient education and counseling.

\section{Patient-specific 3D models for preoperative guidance, planning, and rebearsal of a complex procedure to benefit experienced surgeons}

\section{Preoperative guidance, planning}

The first and most common example for utilizing 3D printing technologies in the surgical field are anatomical organ replicas, printed directly from patients' axial images that portray underlying pathology $(113$, or $71.5 \%$ studies reviewed). These models were used as guides and templates to aid in the preoperative planning of complex surgical procedures (19).

Proponents report that the additional benefits provided from the models include improved decision-making and increased surgeon confidence (in terms of defining the surgical problem, identifying the technical and anatomical aspects of the surgical procedure), which could translate to improved operative outcomes, notably minimizing operative time, reducing blood loss (14) and avoiding complications that could not be anticipated when evaluating $2 \mathrm{D}$ representations (20). Other reports (7) have demonstrated that as many as $30-50 \%$ of surgeons changed their surgical approach after visualizing $3 \mathrm{D}$ models and their potential to restore an aspect of tactile/haptic feedback absent in robotic procedures $(12,21)$. 
Approximately $55.8 \%$ of publications in urology reported surgical planning as a primary outcome of the study, the vast majority focusing on kidney disease surgery, with prostate cancer ( $\mathrm{PCa}$ ) surgeries second most common (20). The frequent use of enhanced, thin slice axial imaging for diagnosis of the disease, combined with the procedure's challenging nature due to complex renal hilar anatomy, unclear depth of tumor invasion, and the potential for renal ischemic damage all make the use of $3 \mathrm{D}$ printing for preoperative planning of nephronsparing surgery in the treatment of suspected renal cell carcinoma an obvious fit. Publications conducted the field of robotic surgery are included in this review, along with a laparoscopic publication that present notable methodology, outcomes or conclusions. Zhang et al., [2016] demonstrated that $3 \mathrm{D}$ printed renal tumor models in preparation for tumor excision demonstrated face (ratings of realism) and content (ratings of educational effectiveness) validity with an effectiveness score of 7.8 and a realism score of 6.0 (on a scale of 1-10). Models were rated highly for their value in surgical planning and training, as well as their ability in portraying resected tumor size (22). However, Zhang et al., did not address specific questions regarding surgical planning or describe how the $3 \mathrm{D}$ printed models impacted specific surgical planning decisions. Wake et al., addressed these questions and demonstrated how presurgical planning decisions regarding nephrectomy type, surgical approach, clamping, and collecting system repair may be impacted with the use of the 3D models (7). Three experienced urologists first reviewed the MRIs alone, followed by the MRIs along with 3D printed models of 10 cases of complex renal cancer (nephrometry score of 6-10). A questionnaire was completed during each session, and the planned surgical approach was evaluated with and without the 3D model, including decisions regarding (I) partial or radical nephrectomy, (II) open or robotic approach, (III) transperitoneal or retroperitoneal approach, and (IV) clamping. The most frequent changes in pre-surgical planning were seen in decisions regarding transperitoneal or retroperitoneal approach and hilar clamping (30-50\%). The authors reported that, although printing the $3 \mathrm{D}$ models from MRI data was feasible, the implementation was both time consuming (mean image post-processing time of 7 hours and mean printing time 10 hours) and costly (\$US 1,000 per kidney tumor case).

Various publications have reported methods for quantitatively validating the accuracy of the $3 \mathrm{D}$ printed models. Michiels et al., was particular notable for verifying the anatomical accuracy of renal tumor models by performing CT scans on the models themselves for comparison with the original imaging. In 16 patients with a median tumor size of $4.7 \mathrm{~cm}$, the difference between the initial CT scan and the $3 \mathrm{D}$ printed kidney was an average of $1.8 \%$ [interquartile range (IQR), $0.8-4.23 \%$ ], $2.35 \%$ (IQR, $0.45-8.6 \%$ ), and $1.8 \%$ (IQR, $1-3.25 \%$ ) for major renal axis, major tumor axis, and median arterial interbranch measurements respectively (23). Dwivedi et $a l$., also created patient-specific, 3D-printed renal tumor molds based on volumetric segmentation of 6 renal masses from multiparametric MRI findings (8). The accuracy of the technology was assessed by adequately fitting the tumor excised during the partial nephrectomy within a 3D printed mold corresponding to the exact size and shape of the tumor. The average cost of printing each mold was \$160 (range, \$20.9-\$350.7).

Komai et al., (9) reported their initial experience with a novel style of 3D-printed kidney, which they called 4D surgical navigation, in 10 patients with renal masses with a RENAL nephrometry score $>8$, who underwent minimally invasive off-clamp partial nephrectomy. The coined term, "4D surgical navigation" was attributed to the modification by which the $3 \mathrm{D}$ printed tumor along with the margin of surrounding healthy parenchymal tissue could be removed. This feature allowed surgeons to preoperatively visualize both pre- and post-tumor resection kidney status, thus the term 4D surgical navigation. In all 10 patients RAPN procedures were successfully completed off-clamp with zero ischemia time and negative surgical margins. Preoperative outcomes reported included a blood loss of $540 \mathrm{~mL}$ in only one case, a postoperative urinary leakage requiring a ureteral stent placement, and no patients requiring a blood transfusion. The 3D-printed tumors together with their margins were nearly identical to the surgical specimens. The cost of creating the current 3D model ranged from \$450-\$680. Despite the high costs, the authors reported that the tactile sensation from touching the $3 \mathrm{D}$ printed kidney also allowed the surgeons to more fully understand the kidney anatomy, especially the interrelationship among the kidney, tumor, and vasculature.

3D technology has also been tested in the field of PCa. High accuracy ratings for $3 \mathrm{D}$ printed models utilized as a surgical planning and navigation tool for genitourinary malignancies have been reported by Porpiglia et al., during a live surgical event that included eight robot-assisted radical prostatectomies (RARPs) for organ-confined PCa. A total of 144 participants shared in discussions with the surgeon 
utilizing hand-held 3D printed prostate replicas prior to the live surgery. Participants then completed a questionnaire that included subjective questions rating the accuracy and usefulness of the 3D printed models. Satisfactory results were obtained regarding both surgical planning (from 7 to $9 / 10)$ and anatomical accuracy $(10 / 10)$. The urologists who included both the live surgeons and the attendants expressed high satisfaction with the printed models (10). Shin and colleagues, reported that combining MRI and $3 \mathrm{D}$ models can aid with identification of precise tumor location, as identified by multi-parametric MRI (mpMRI), and its relationships with neurovascular bundles (NVBs) and the prostate capsule (11). This approach allowed the surgeon to modulate the procedure and the nerve-sparing technique with improved confidence and precision while preserving oncological effectiveness. Chandak et al., also highlighted the critical value of using $3 \mathrm{D}$ printed models for appreciating the spatial relationships of the NVB in planning and performing nerve-sparing prostatectomies (12). Surgeons in this study reported that interacting with this $3 \mathrm{D}$ printed model during the robotic procedure restored an aspect of tactile feedback lost in robotic surgery, allowing the surgeon to perform incremental nerve spare or wider excision of the NVBs around the palpable tumors optimizing outcomes with high patient satisfaction $(11,12)$. Minimal research has been performed with 3D printing in the arena of preoperative planning prior to renal transplantation. However, this will soon become a promising area of future application with the increasing adoption of the robot-assisted approach in both donor and recipient renal transplantation (24).

\section{Surgical rehearsal}

The aforementioned $3 \mathrm{D}$ printed models utilized conventional 3D-printing techniques, which have limited capability to generate a high-fidelity model with tissue-like properties suitable for a true hands-on surgical simulation and preoperative case rehearsal. This is a consequence of a lack of available printing polymers that can reproduce human tissue properties. Furthermore, available polymers which can be easily adapted to mimic human tissue properties are challenging to constitute into printing material. To overcome these limitations $3 \mathrm{D}$ printing was combined with polymer casting to create models from silicone, wax, and/or hydrogels (25). These materials have the inherent ability to approximate mechanical properties of living organs.

The majority of existing publications utilizing this fabrication method were mainly geared towards laparoscopic surgery, though this is beginning to shift $(26,27)$. Von Rundstedt's robotic surgical rehearsals using rapidly produced, realistic silicone models entailed 10 patient-specific models, fabricated to duplicate their complex tumor anatomy (average RENAL nephrometry scores of 8.9 and mean maximal tumor diameter of $40.6 \mathrm{~mm}$ ). The mean resection times between model and patient (6:58 vs. 8:22 $\mathrm{min}, \mathrm{P}=0.162)$ and tumor volumes extrapolated from the computer model, excised model, and excised tumor (38.88 vs. 38.50 vs. $41.79 \mathrm{~mm}^{3}, \mathrm{P}=0.98$ ) were not significantly different. The authors argue this demonstrates value in assessing the feasibility of RAPN within an acceptable total ischemia time and its help in determining if the operation can/should be done at all. Ghazi et al., developed a personalized kidney model with five lesions representing the vascular ramifications in patients with von Hippel-Lindau (VHL) syndrome. These models were tested in surgical rehearsals to assess the feasibility of a RAPN under ischemic conditions (13). The procedure was successfully completed with excision of all five tumors with negative surgical margins and an ischemia time of less than 23 minutes. The authors attribute this success to the ability to attempt several rehearsals with the kidney perfused through which various approaches to resecting the tumors (including resecting two of the smaller tumors off clamp prior to inducing warm ischemia), the ability to examine the models following each simulation which enabled the surgeon to modify the approach accordingly, and the model's ability to predict the surgical outcome. Expanding beyond von Rundstedt's rapidly produced, realistic silicone models, this developed platform simulated kidney texture, anatomy, and perfusion, incorporating the surrounding perinephric fat, bowel and musculature, including bleeding vessels simulating bleeding during tumor resection allowing for a full-immersion experience (Figure 1). Maddox et al. 2018 quantified the benefit of pre-op rehearsals on patient outcomes using specific 3D kidney models. The seven models fabricated were constructed using a resin suitable for surgical resection (agarose gel solution) to create a spongy texture with color variation to delineate the renal parenchyma, tumor, vascular structures, components of the collecting system, and the proximal ureter. A simulation using the da Vinci platform was completed prior to RAPN. Outcomes from this study reported lower ischemic time, fewer positive surgical margins, shorter hospitalization, fewer post-operative complications, and 

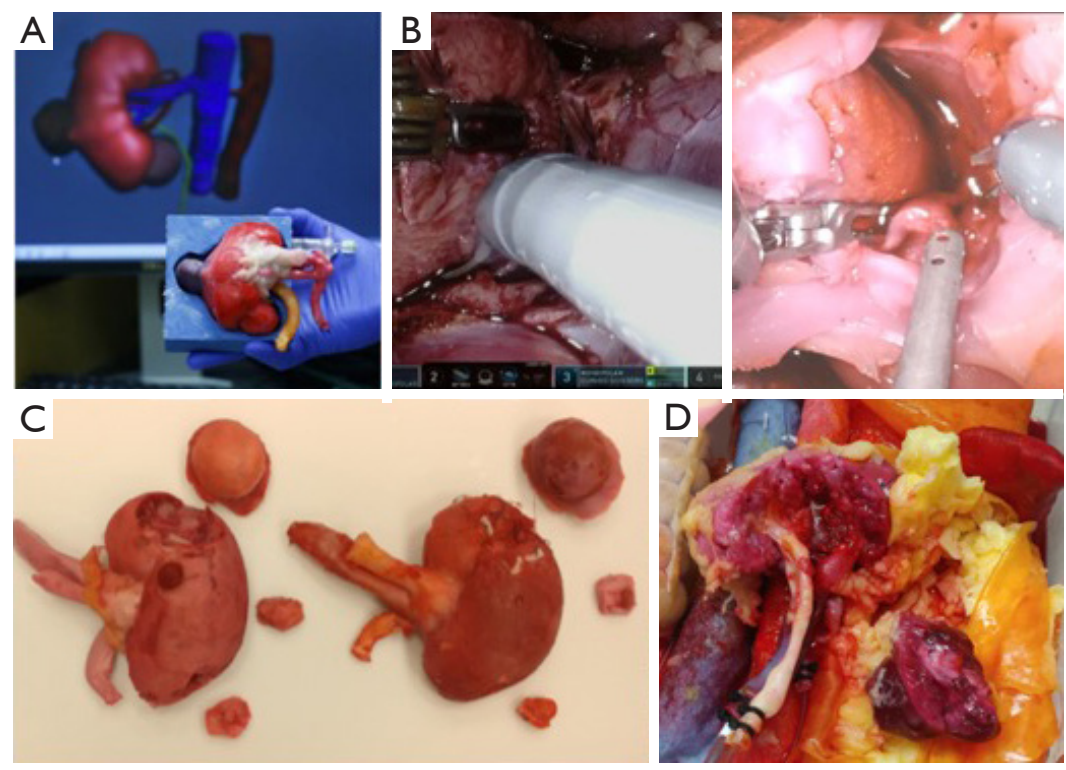

Figure 1 Patient-specific simulation. (A) Computer design resulting from segmentation of the patients CT scan in the background with personalized kidney model in its cast; (B) excision of a tumor with bleeding (left live surgery, right simulated rehearsal); (C,D) examination of the specimen following simulated rehearsals showing the excised lesions.

lower estimated blood loss in patients which the team performed pre-operative surgical rehearsals. However, the only significant difference found compared to case controls was lower blood loss (186 vs. $236 \mathrm{~mL}$, $\mathrm{P}=0.01)(14)$.

Despite the small cohort and low statistical power of these studies, these results contribute to the growing body of evidence supporting the efficacy of 3D printed simulations prior to complex surgery. As the field continues to evolve, we may indeed see the prediction postulated by Manning et al. 2018 come true: "a 'practice before you play' model for complicated surgery. The addition of surgical rehearsal may eventually add to the standard of care, even for experienced urologists" (28).

\section{D generic procedural models for surgical training (bands- on surgical practice) for novice surgeons}

One of the most pressing applications of $3 \mathrm{D}$ printing for surgical education is the fabrication of simulation-based training models. The training of inexperienced surgeons, especially residents, outside the operating room is essential in light of ACGME work hour restrictions, expensive operating room time, and increased emphasis on patient safety (29). Simulators enable learners to practice psychomotor, technical, and judgment skills to proficiency in a setting that does not cause harm to patients (30). 3D printing technology provides the opportunity for novices to practice with flexible hours using both standardized models for evaluation purposes in urology as well as rare complications and anatomical variations that they may not have the opportunity to see during their training program (20).

$3 \mathrm{D}$ printed models have been utilized as a training platform with an implicit application as simulation training tools for the acquisition of technical skills in laparoscopic and endoscopic procedures (3). The literature supporting the value of $3 \mathrm{D}$ models for practice of technical and procedural skills in urological robotic procedures is limited but rapidly growing. Uwechue et al., demonstrated the value of a hybrid $3 \mathrm{D}$-printed simulation model for roboticassisted kidney transplantation combining a $3 \mathrm{D}$ printedmoulage, integrated with deceased donor vessels (15). The prosthetic component consists of a synthetic $3 \mathrm{D}$ printed cradle of an anatomically accurate pelvis printed in life size, a kidney without vessels, and a plinth to support the kidney in the correct operative position within the pelvis. The da Vinci Si robotic system was successfully docked in situ due to lack of an abdominal wall or box trainer. Two robotic surgeons performed vascular anastomoses between the cadaveric donor renal vessels and cadaveric iliac vessels, splinting the $3 \mathrm{D}$ printed recipient iliac vessels using 6.0 prolene sutures. Mean anastomotic procedural time was 

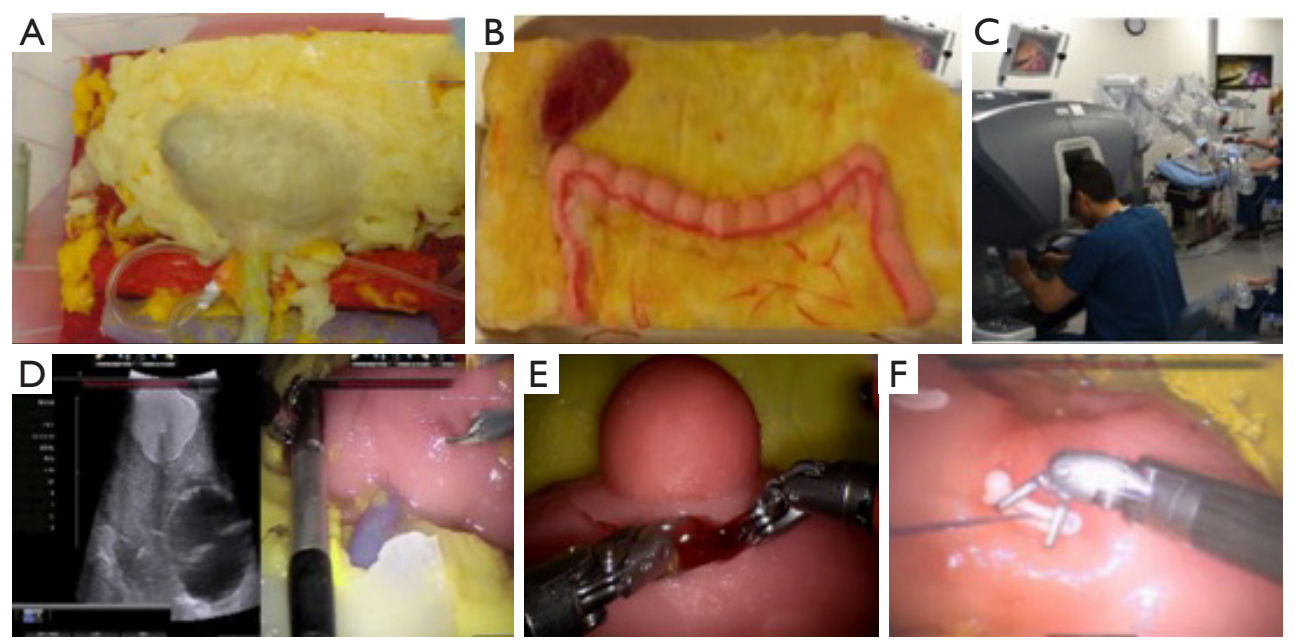

Figure 2 Validation of the simulation platform for RAPN. (A) Left kidney hydrogel phantom, encompassing vascular and urine channels for perfusion; (B) simulation platform for RAPN,; including kidney (containing renal hilar vessels, PCS and tumors), major abdominal vessels, perinephric fat, posterior abdominal muscles, spleen and overlying bowel; (C) full immersion simulation on the procedural platform in an operating room; (D) intraoperative ultrasound of the simulated tumor (left) with probe on model after dissection of Gerota's fascia (right); (E) simulated excision of tumor with functional bleeding; (F) simulated closure of the parenchymal defect, demonstrating sliding clip renorrhaphy after tumor resection. RAPN, robot-assisted partial nephrectomy; PCS, pelvicalyceal system.

20 min per vessel. Vascular anastomotic patency was tested by intravascular injection of saline using a hypodermic needle. Good anastomotic patency and leak-resistance was demonstrated in the simulations. The purpose of the model was to optimize the surgeons' training in vascular anastomoses in a timed and reproducible manner to allow objective assessment of their competencies.

Ghazi et al., validated a high-fidelity, full-task trainer for RAPN within a full-immersion simulation environment (16). They created a simulated inanimate model composed of hydrogel polymer [polyvinyl alcohol (PVA)], using a patient's computerized tomography scan with a $4.2 \mathrm{~cm}$, upper pole renal tumor (RENAL score $7 x$ ) to construct an idealized tumor-laden kidney with a patent pelvicalyceal system (PCS) and hollow, watertight vasculature that were registered in $3 \mathrm{D}$ printed kidney molds. To replicate the entire procedure, the hydrogel kidney was layered in its anatomical configuration within a body cast that included posterior abdominal musculature, perinephric fat, and relevant organs (liver, spleen and colon). The organs were layered in their anatomical position to preserve their spatial relationships, and a final processing cycle generated cohesion of all the structures to replicate the connective tissue attachments between intra-abdominal organs. The simulation platform demonstrated excellent face and content validity, providing a complete tool for improving and evaluating surgical skills before hands-on exposure (Figure 2).

The same group developed a platform for robotic radical prostatectomy simulation. Anatomically accurate models of the human pelvis, bladder, prostate, urethra, NVBs, and relevant adjacent structures were created from patients' MRIs using PVA hydrogels and 3D-printed injection molds. Bladder neck dissection, seminal vesicle mobilization, nervesparing prostatectomy, and urethra-vesical anastomosis (UVA) were simulated by five experts (>500 caseload) and 9 novices (<50 caseload) (Figure 3). The authors sought to validate incorporated Clinically-relevant Performance Metrics of Simulation (CRPMS) into this hydrogel model for nerve-sparing RARP (NS-RARP) (nerve tension during NVB dissection, surgical margins, and UVA integrity) and correlate these metrics to standardized objective assessments of performance (GEARS and RACE). Experts achieved faster task specific times for nerve sparing $(\mathrm{P}=0.007)$ and superior margin status $(\mathrm{P}=0.011)$. Nerve forces applied during the simulation, measured by calibrated stretch sensors incorporated into the NVBs, were significantly lower for experts. Higher force sensitivity (Subcategory of GEARS Score) and Total GEARS Score correlated with lower nerve forces applied with total energy (J) -0.66 (0.019) and $-0.87(0.000)$, respectively, which was significantly different between novices and experts $(\mathrm{P}=0.003)$. UVA leak rate highly correlated with total RACE score -0.86 (0.000), 

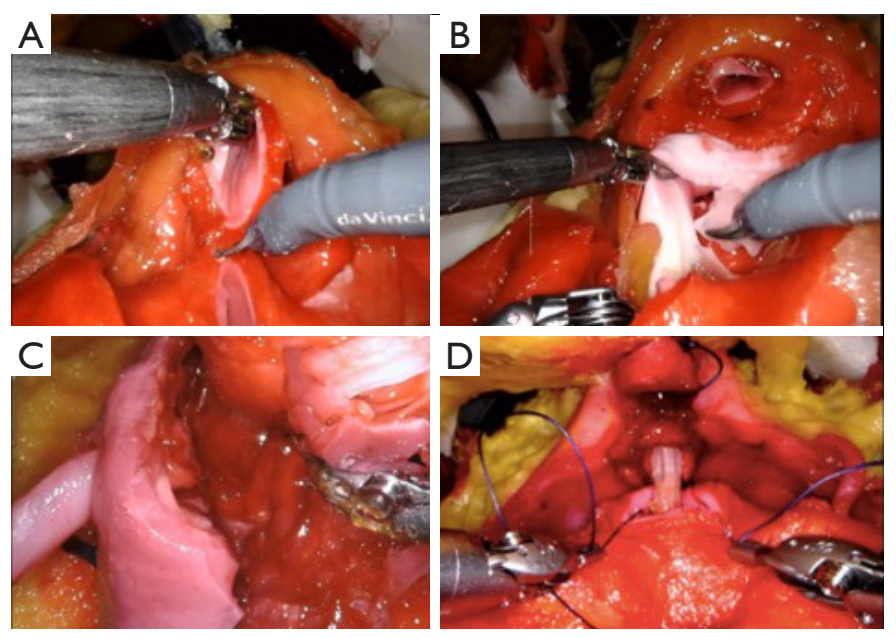

Figure 3 NS-RARP simulation tasks. (A) bladder neck dissection; (B) seminal vesicle mobilization; (C) left nerve-sparing prostatectomy; (D) UVA. NS-RARP, nerve-sparing robot-assisted radical prostatectomy; UVA, urethrovesical anastomosis.
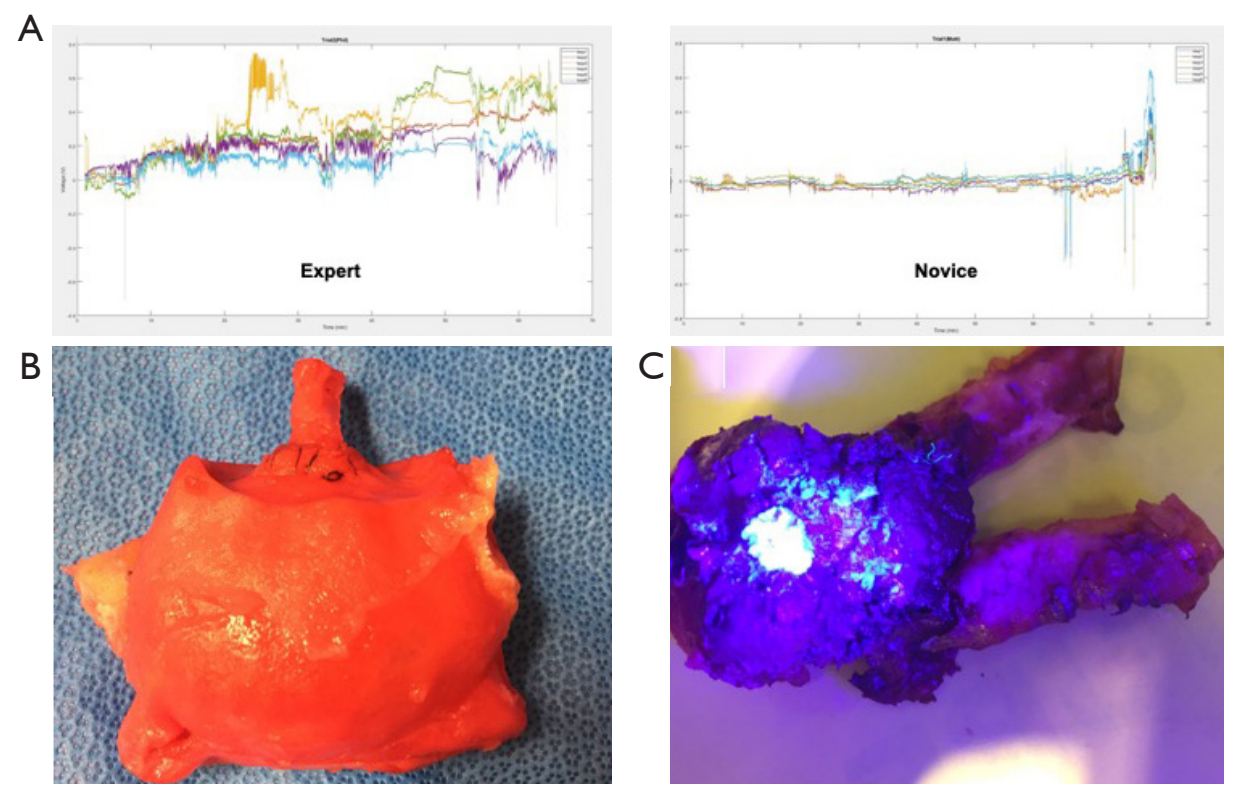

Figure 4 CRPMS incorporated into the NS-RARP simulation tasks. (A) Qualitative representation of NVB tensile forces applied by novices and experts during NS-RARP simulation; (B) example of the post-simulation UVA that demonstrated no leak; (C) example of prostate margins after NS-RARP simulation under UV light. CRPMS, clinically-relevant performance metrics of simulation; NS-RARP, nervesparing robot-assisted radical prostatectomy; NVB, neurovascular bundle; UVA, urethrovesical anastomosis.

which was also significantly different between novices and experts $(\mathrm{P}=0.003)$ (Figure 4). This study presents a novel method for real-time assessment and feedback during robotic surgery training utilizing incorporated CRPMS into hydrogel models fabricated using 3D printing and hydrogel molding technology (17).
These efforts in combining 3D printing technology with other transformative technologies has provided a platform for reproducing realistic procedural models that have the ability to accurately portray anatomical characteristics, reproducing tissue characteristics, and replicating the entire gestalt of the operative experience, but with the added 
capacity to provide objective procedural metrics that permit real-time feedback and assessment during surgical training.

In addition to realistic polymer-based $3 \mathrm{D}$ models permitting trainees to acquire and practice the surgical and technical steps for an entire procedure before working on a patient, hard 3D models also have significant value for increasing knowledge for medical students. Silberstein and Knoedler recruited $1^{\text {st }}$ year med students with limited knowledge of anatomy and imaging (i.e., patients) to review five $3 \mathrm{D}$ printed renal tumor models scheduled for a RAPN. The authors proposed that accelerating the medical student learning curve can be helpful given the increasing amount of knowledge expected to be learned by students in a short amount of time. Unfortunately, the only evidence presented was the ability of the medical students to reference anatomical landmarks intraoperatively when questioned by the surgeons. They also demonstrated that $1^{\text {st }}$ year medical students and residents were more able to accurately characterize renal tumors (via assignment of RENAL nephrometry scores) when given $3 \mathrm{D}$ printed models as compared to only having 2D cross-sectional images in 3 of the 4 components of RENAL nephrometry scores (radius, nearness to the urine collecting system, and location). Interestingly when compared to expert urologists, the interrater agreement (reliability) improved with the $3 \mathrm{D}$ printed models $(\mathrm{P}=0.002)$, thus demonstrating an improvement in the learning curve required to understand complex renal tumor anatomy (18). Similarly, a study of 3D printed prostate models segmented from PCa patients' MRIs improved medical students' ability to accurately identify the location of the cancer focus compared to MRI alone. The students demonstrated an improvement in accuracy by $17 \%$ when aided by $3 \mathrm{D}$ models compared to MRI alone, which is an arguably more difficult imaging modality to interpret (31). Marconi et al., found that 3D printed models of fifteen patients scheduled for laparoscopic nephrectomy assisted medical students, surgeons, and radiologists in identifying anatomic structures more quickly and accurately. The less experienced medical students perceived the highest benefit $(53.9 \% \pm 4.14 \%$ of correct answers with $3 \mathrm{D}$-printed models), followed by surgeons and radiologists. Assessment time was almost $50 \%$ shorter $(60.67 \pm 25.5 \mathrm{~s})$ than reviewing corresponding conventional CT scans (127.04 $35.91 \mathrm{~s})$ (32).

\section{Patient education and counseling}

The importance of patient understanding their conditions, treatment options, and planned procedures is often underappreciated. This is critical for ethical reasons, as an awareness of these key features is necessary to have truly informed consent, but also as a matter of professionalism, in that physicians should engage in good-faith shared decision-making with their patients with both parties as well-informed as possible. This can be especially important in cases such as incidentally found, asymptomatic renal masses, where misunderstanding can lead to poor decisionmaking. The current surgical counseling/planning approach involves the use of pictorial images, diagrams, and 2D imaging, which are ineffective in depicting patient-specific intricacies, resulting in a conceptual challenge. Patients and surgeons, holding an exact 3D replica of their organs, have a better understanding of their illness, leading to more informed treatment decisions a deeper understanding of the procedure, the alternative approaches, technical details, and possible complications or pitfalls.

The use of $3 \mathrm{D}$ models as a patient education tool has been evaluated both qualitatively and quantitatively, with consistently positive impacts. Porpiglia et al., tested the validity of virtually rendered $3 \mathrm{D}$ printed models, used before robot-assisted prostatectomy for $\mathrm{PCa}$ and nephron-sparing surgeries during a live surgical symposium. A purpose-built face and content validity survey composed of open-ended questions with a 10 -point ordinal Likert scale was implemented. All 18 patients reported favorable feedback (all scores of 9 out of 10 or better) about the use of this technology during discussion of their case with the surgeon (10). Silberstein $e t a l$., noted that 5 patients and families with complex renal tumors scheduled for RAPN verbally expressed improved comprehension of the size, location, and the intended surgical intervention after viewing their $3 \mathrm{D}$ printed renal models. The same team completed a follow-up study that focused on demonstrating patient understanding and satisfaction with $3 \mathrm{D}$ printed replicas of their kidneys and pathology prior to RAPN (5). Patients elicited a superior understanding of kidney physiology, anatomy, tumor characteristics, and planned surgical intervention when compared to traditional education using CT images, as determined by standardized survey scores. After viewing their personal 3D kidney model, patients demonstrated an improvement in understanding of basic kidney physiology by $16.7 \%$, kidney anatomy by $50 \%$, tumor characteristics by $39.3 \%$, and the planned surgical procedure by $44.6 \%$ compared to patients without this visual aid. Patients reported a higher overall satisfaction in their surgical experience, which was attributed to an 
improved understanding of their disease and surgical intervention (18). Lastly, Zhang et al., with 10-point rating scale for the models where 10 is very useful/very realistic/ excellent, reported an average score of $\geq 9.0$ from patients. The authors argued it can improve health literacy in patients with limited knowledge their disease (22).

Health literacy is an individual patient's ability to obtain, process, and understand health information and services needed to make appropriate health decisions. It has been shown that health literacy plays an important role in quality health care, and patients with better ability to process health information have better outcomes. It is apparent that $3 \mathrm{D}$ printed models are effective tools that would result in patients with a better understanding of their illness, leading to more informed treatment decisions and a betterinformed consent process.

\section{Conclusions/discussion/future directions}

3D modeling has emerged as a novel, exciting, and effective tool in the hands of patients, trainees, and even experienced surgeons. In the last 5 years, it's specific applications in the field of urology have been explored widely, with promising results. As an educational vehicle, it has particular value for augmenting and improving the training of novice surgeons honing basic techniques in a safe environment. Its value as a pre-operative planning tool has been especially impactful in robotic partial nephrectomies and radical prostatectomies, refining the approach, saving valuable operative time, and increasing the surgeon confidence. Lastly, it has received a long-awaited welcome from patients and students as a modality that can significantly improvement their comprehension of the anatomy, complexities of surgical conditions, and the procedures being offered.

The limitations existing in the current literature are similar to other novel applications, generally small sample sizes with short term follow-up and a lack of level one evidence. Furthermore, the lack of technical standardization (file formats, software used, and accessibility of technology), absence of control groups, and varying cost estimates limits a head to head comparison of the available studies. Despite these limitations, the technology remains widely accepted with some surgeons even reporting a cognitive benefit that would be difficult to quantify (33). Randomized prospective studies are required to truly evaluate the tangible benefits of this technology and to quantify the added value.

The current barriers to the adoption or this technology into standard urological surgery practice are manifold, but solutions are in continuous development. The primary concern is cost, which is driven by the choice of materials and technique, availability of modeling software, and access to a $3 \mathrm{D}$ printer. As with any new technology, the initial highly variable costs remain expensive but with increased availability of low-cost $3 \mathrm{D}$ printers and open source modeling software this technology is becoming an established standard at many academic centers. However, not all printers are equal. A single head fused deposition modeling (FDM) printer that can only print a single material of limited dimensions with lengthy print times, costs $<\$ 1,000$. On the other hand, a six head SLA printer can print various materials within the same print of varying mechanical properties on a larger print bed with significantly shorter print times, but costs can exceed $\$ 300,000$. The tradeoff between costs and benefits necessitates a close inspection of the true value gained. In monetary terms, for example, does 10 minutes saved in an operating room, $100 \mathrm{~mL}$ less blood loss, or better patient satisfaction have the same value as 1 hour of work on the 3D model design or its production? Current estimates have proven the notion that the cost of the model may easily be offset by the added benefit of reduced operative times alone. In the study by Komai et al., they reported that using the $3 \mathrm{D}$ printed kidney significantly shortened the duration of intraoperative ultrasound (mean $3.3 \mathrm{~min}$ ) with a $3 \mathrm{D}$ model compared to a retrospective matched cohort without the 3D model (6.3 min, $\mathrm{P}=0.021)$ (9). With an average cost of operative time pegged at $\$ 62$ a minute, the costs savings from this minor step (\$186) alone would cover almost half the costs of the 3D model (\$450-\$680) (34).

Other remaining challenges include issues of biocompatibility between 3D printing materials, and lack of regulatory policies. Some regulatory bodies are currently under consultation for frameworks regarding the implications for the use of $3 \mathrm{D}$ printing in healthcare. A recently announced FDA publication of a 31-page set of guidelines for manufacturers producing medical products via $3 \mathrm{D}$ printing/ additive manufacturing has been published. Although this information is presented as non-binding recommendations, the agency is the first to provide such a comprehensive regulatory framework (35). With continued research and development, increased funding, and greater popularity, the process will become quicker, cheaper, and more accessible.

The future of $3 \mathrm{D}$ printing in urology is bright. It may represent the next revolution in medical education, surgical practice, and surgical rehearsals. 3D printing files/databases may eventually replace medical textbook illustrations. Augmented reality and telesurgery/telementoring are on the horizon, creating the possibility of surgical overlays onto 
the operating field, the transmission of real-time images onto head-mounted displays and remote coaching from an experienced surgeon may soon be a reality (21).

In urology specifically, 3D modeling will expand into new procedures, such as preoperative planning for brachytherapy, development of artificial sphincters, and eventually the production of printed kidneys for transplant and bladders post-cystectomy. It seems that the opportunities for innovation in robotic education with $3 \mathrm{D}$ printing technology are boundless, and we are currently in the middle of a dynamic, paradigm-shifting era.

\section{Acknowledgments}

Funding: Dr. Ghazi reports grants from Intuitive Surgical and personal fees from Olympus America.

\section{Footnote}

Provenance and Peer Review: This article was commissioned by the Guest Editor (Ashok K. Hemal) for the series "Robotic-assisted Urologic Surgery" published in Translational Andrology and Urology. The article was sent for external peer review organized by the Guest Editor and the editorial office.

Conflicts of Interest: All authors have completed the ICMJE uniform disclosure form (available at http://dx.doi. org/10.21037/tau.2020.01.03). The series "Robotic-assisted Urologic Surgery" was commissioned by the editorial office without any funding or sponsorship. AEG reports consulting fees from Olympus America, personal fees from Johnson \& Johnson, grants from Intuitive Surgical; a patent Systems, Models and Methods for simulating Surgery on Anatomical Organs pending. BAT has no other conflicts of interest to declare.

Ethical Statement: The authors are accountable for all aspects of the work in ensuring that questions related to the accuracy or integrity of any part of the work are appropriately investigated and resolved.

Open Access Statement: This is an Open Access article distributed in accordance with the Creative Commons Attribution-NonCommercial-NoDerivs 4.0 International License (CC BY-NC-ND 4.0), which permits the noncommercial replication and distribution of the article with the strict proviso that no changes or edits are made and the original work is properly cited (including links to both the formal publication through the relevant DOI and the license). See: https://creativecommons.org/licenses/by-nc-nd/4.0/.

\section{References}

1. Martelli N, Serrano C, van den Brink H, et al. Advantages and disadvantages of 3-dimensional printing in surgery: a systematic review. Surgery 2016;159:1485-500.

2. Auricchio F, Marconi S. 3D printing: clinical applications in orthopaedics and traumatology. EFORT Open Rev 2017;1:121-7.

3. Parikh N, Sharma P. Three-dimensional printing in urology: history, current applications, and future directions. Urology 2018;121:3-10.

4. Malik HH, Darwood AR, Shaunak S, et al. Threedimensional printing in surgery: a review of current surgical applications. J Surg Res 2015;199:512-22.

5. Silberstein JL, Maddox MM, Dorsey P, et al. Physical models of renal malignancies using standard crosssectional imaging and 3-dimensional printers: a pilot study. Urology 2014;84:268-72.

6. von Rundstedt FC, Scovell JM, Agrawal S, et al. Utility of patient-specific silicone renal models for planning and rehearsal of complex tumour resections prior to robotassisted laparoscopic partial nephrectomy. BJU Int 2017;119:598-604.

7. Wake N, Rude T, Kang SK, et al. 3D printed renal cancer models derived from MRI data: application in pre-surgical planning. Abdom Radiol (NY) 2017;42:1501-9.

8. Dwivedi DK, Chatzinoff Y, Zhang Y, et al. Development of a patient-specific tumor mold using magnetic resonance imaging and 3-dimensional printing technology for targeted tissue procurement and radiomics analysis of renal masses. Urology 2018;112:209-14.

9. Komai Y, Sugimoto M, Gotohda N, et al. Patient-specific 3-dimensional printed kidney designed for "4D" surgical navigation: a novel aid to facilitate minimally invasive off-clamp partial nephrectomy in complex tumor cases. Urology 2016;91:226-33.

10. Porpiglia F, Bertolo R, Checcucci E, et al. Development and validation of $3 \mathrm{D}$ printed virtual models for robotassisted radical prostatectomy and partial nephrectomy: urologists' and patients' perception. World J Urol 2018;36:201-7.

11. Shin T, Ukimura O, Gill IS. Three-dimensional printed model of prostate anatomy and targeted biopsy-proven index tumor to facilitate nerve-sparing prostatectomy. Eur Urol 2016;69:377-9.

12. Chandak P, Byrne N, Lynch H, et al. Three-dimensional 
printing in robot-assisted radical prostatectomy - an idea, development, exploration, assessment, long-term followup (IDEAL) phase 2a study. BJU Int 2018;122:360-1.

13. URMC. Practice makes perfect -- fake organs guide the way for 'impossible' cancer surgery. Available online: https://www.urmc.rochester.edu/news/story/5366/ practice-makes-perfect----fake-organs-guide-the-way-forimpossible-cancer-surgery.aspx

14. Maddox MM, Feibus A, Liu J, et al. 3D-printed soft-tissue physical models of renal malignancies for individualized surgical simulation: a feasibility study. J Robot Surg 2018;12:27-33.

15. Uwechue R, Gogalniceanu P, Kessaris N, et al. A novel 3D-printed hybrid simulation model for roboticassisted kidney transplantation (RAKT). J Robot Surg 2018;12:541-4.

16. Ghazi A, Stone J, Candela B, et al. Simulated inanimate model for physical learning experience (simple) for robotic partial nephrectomy using a $3 \mathrm{~d}$ printed kidney model. J Urol 2015;193:e778.

17. Witthaus M, Campbell T, Melnyk R, et al. V08-06 Validation of a full-immersion simulation platform with performance metrics for robotic radical prostatectomy (RARP) using three-dimensional printing and hydrogel molding technology. J Urol 2019;201:e849-50.

18. Knoedler M, Feibus AH, Lange A, et al. Individualized physical 3-dimensional kidney tumor models constructed from 3-dimensional printers result in improved trainee anatomic understanding. Urology 2015;85:1257-61.

19. Aimar, A, Palermo A, Innocenti B. The role of $3 \mathrm{D}$ printing in medical applications: a state of the art. J Healthc Eng 2019;2019:5340616.

20. Cacciamani GE, Okhunov Z, Meneses AD, et al. Impact of three-dimensional printing in urology: state of the art and future perspectives. a systematic review by ESUTYAUWP group. Eur Urol 2019;76:209-21.

21. Westerman ME, Matsumoto JM, Morris JM, et al. Three-dimensional printing for renal cancer and surgical planning. Eur Urol Focus 2016;2:574-6.

22. Zhang Y, Ge HW, Li NC, et al. Evaluation of threedimensional printing for laparoscopic partial nephrectomy of renal tumors: a preliminary report. World J Urol 2016;34:533-7.

23. Michiels C, Jambon E, Bernhard JC. Measurement of the accuracy of 3D-printed medical models to be used for robot-assisted partial nephrectomy. AJR Am J Roentgenol 2019;213:626-31.

24. Hameed AM, Yao J, Allen RDM, et al. The evolution of kidney transplantation surgery into the robotic era and its prospects for obese recipients. Transplantation 2018;102:1650-65.

25. Smektala T, Golab A, Krolikowski M, et al. Low cost silicone renal replicas for surgical training - technical note. Arch Esp Urol 2016;69:434-6.

26. Kusaka M, Sugimoto M, Fukami N, et al. Initial experience with a tailor-made simulation and navigation program using a 3-D printer model of kidney transplantation surgery. Transplant Proc 2015;47:596-9.

27. Golab A, Smektala T, Kaczmarek K, et al. Laparoscopic partial nephrectomy supported by training involving personalized silicone replica poured in three-dimensional printed casting mold. J Laparoendosc Adv Surg Tech A 2017;27:420-2.

28. Manning TG, O'Brien JS, Christidis D, et al. Three dimensional models in uro-oncology: a future built with additive fabrication. World J Urol 2018;36:557-63.

29. Reznick RK and MacRae H. Teaching surgical skillschanges in the wind. N Engl J Med 2006;355:2664-9.

30. Badash I, Burtt K, Solorzano CA, et al. Innovations in surgery simulation: a review of past, current and future techniques. Ann Transl Med 2016;4:453.

31. Ebbing J, Jäderling F, Collins JW, et al. Comparison of $3 \mathrm{D}$ printed prostate models with standard radiological information to aid understanding of the precise location of prostate cancer: a construct validation study. PLoS One 2018;13:e0199477.

32. Marconi L, Desai MM, Ficarra V, et al. Renal preservation and partial nephrectomy: patient and surgical factors. Eur Urol Focus 2016;2:589-600.

33. Porpiglia F, Amparore D, Checcucci E, et al. Current use of three-dimensional model technology in urology: a road map for personalised surgical planning. Eur Urol Focus 2018;4:652-6.

34. Childers CP, Maggard-Gibbons M. Understanding costs of care in the operating room. JAMA Surg 2018;153:e176233

35. U.S. Department of Health and Human Services, Food and Drug Administration, Center for Devices and Radiological Health, Center for Biologics Evaluation and Research. Technical Considerations for Additive Manufactured Medical Devices: Guidance for Industry and Food and Drug Administration Staff. 2017. Available online: https://www.fda.gov/media/97633/download

Cite this article as: Ghazi AE, Teplitz BA. Role of 3D printing in surgical education for robotic urology procedures. Transl Androl Urol 2020;9(2):931-941. doi: 10.21037/tau.2020.01.03 
Table S1 Studies retrieved from literature research reporting the impact of 3D printing for education in the field of urology $\begin{array}{llll}\text { Author } & \text { Year No of cases Target organ Disease pathology Robotic procedure }\end{array}$

Patient-specific 3D models for guidance, planning, and rehearsal of a complex procedure to benefit practicing surgo

3D printer Material

Outcomes

\begin{abstract}
Silberstein et al. (5) $\quad 2014$
Partial nephrectomy
\end{abstract}

Wake et al. (7) 2017

$2017 \quad 10$

Kidney $\quad$ Renal concer

NA

Translucent resin wit
color coded tumor

\begin{tabular}{|c|c|c|c|c|c|c|}
\hline Dwivedi et al. (8) & 2018 & 6 & Kidney & Renal cancer & Partial nephrectomy & Projet 3512HD \\
\hline Komai et al. (9) & 2016 & 10 & Kidney & Renal cancer & Partial nephrectomy & Objet 500 Connex 3 \\
\hline Porpiglia et al. (10) & 2018 & 18 & $\begin{array}{l}10 \text { kidneys; } \\
8 \text { prostates }\end{array}$ & Kidney and PCa & $\begin{array}{l}\text { Partial nephrectomy; radical } \\
\text { prostatectomy }\end{array}$ & NA \\
\hline Shin etal. (11) & 2016 & 5 & Prostate & PCa & $\begin{array}{l}\text { Nerve sparing radical } \\
\text { prostatectomy }\end{array}$ & Formlab 1 \\
\hline Chandak et al. & 2018 & 10 & Prostate & $\mathrm{PCa}$ & $\begin{array}{l}\text { Nerve sparing radical } \\
\text { prostatectomy }\end{array}$ & Objet 500 Cont \\
\hline
\end{tabular}

$\begin{array}{lccccc}\begin{array}{l}\text { von Rundstedt } \\ \text { et al. (6) }\end{array} & 2017 & 10 & \text { Kidney } & \text { Kidney cancer } & \text { Partial nephrectomy } \\ \text { Ghazi et al. (13) } & 2018 & 1 & \text { Kidney } & \text { VHL syndrome } & \text { Partial nephrectomy } \\ \text { Maddox et al. (14) } & 2018 & 10 & \text { Kidney } & \text { Kidney cancer } & \text { Partial nephrectomy }\end{array}$

FDM printer$$
\text { 3D generic procedural models for surgical training (hands-on surgical practice) for novice surgeons }
$$

$\begin{array}{llllll}\text { Uwechue et al. (15) } 2018 \quad 2 \text { models } & \text { Kidney } & \text { Renal transplant } & \text { Renal transplant (recipient) } & \begin{array}{l}\text { Hybrid model (with cadaveric } \\ \text { donor and iliac vessels and } 3 D\end{array}\end{array}$ printed kidney, bony pelvis)

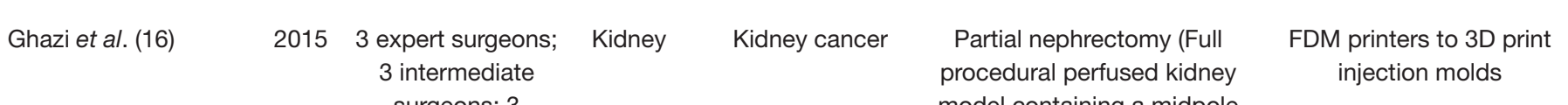 $\begin{array}{cc}3 \text { intermediate } & \text { procedural perfused kidney } \\ \text { surgeons; } 3 & \text { model containing a midpole }\end{array}$ tumor and surrounding organs

\begin{tabular}{|c|c|c|c|c|c|}
\hline Witthaus et al. (17) & 2019 & $\begin{array}{c}14(5 \text { experts \& } 9 \\
\text { novices) }\end{array}$ & Prostate & $\mathrm{PCa}$ & 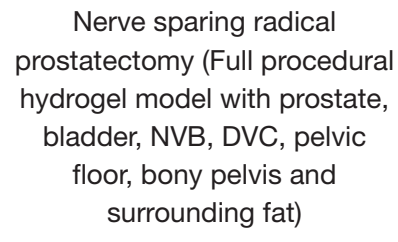 \\
\hline
\end{tabular}

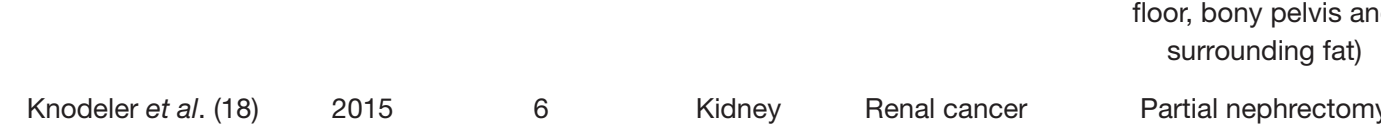
$\begin{aligned} & \text { Clear, transparent } \\ & \text { flexible material }\end{aligned}$
Study group consisted of 3 experts that reviewed MRl followed MRI $+3 \mathrm{D}$ model to identify
changes in surgical approach based on both modalities

NA

Radio-pathological co-localization of in vivo quantitative mpMRI features with ex vivo surgical
specimens of patients with renal mases usint patient-specific $3 D$-printed tumor molds

Case series to demonstrate the impact of $3 \mathrm{D}$ patient models utilizing $4 \mathrm{D}$ surgical navigation in

$$
\text { facilitating off-clamp partial nephrectomy }
$$

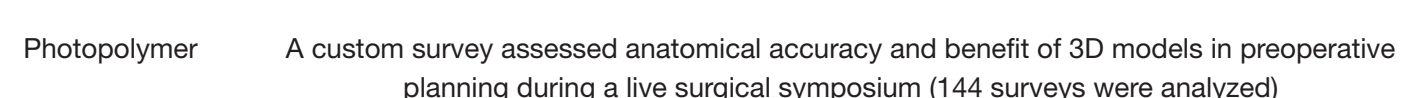

A custom survey assessed anatomical accuracy and benefit of $3 \mathrm{D}$ models in preoperative

undeasibility of life-size 3D printed prostate model as a reference tool (facilitating the understanding of proximity of the index cancer to the prostate capsule and nerve bundles)

Veroclear fullcure Single surgeon, case series of 10 radical prostatectomies performed using $3 D$ printed models as a preoperative planning tod

Silicone Single surgeon case series ffeasibility study) in 10 patients with complex renal tumors in which To confirm accuracy of the models a volumetric comparison between tumor diameter in the
. model and excised specimen was performed.

Hydrogel (PVA) Case report demonstrating the feasibility of patient-specific surgical rehearsals in a VH-

hotopolymer (agarose Case series (feasibility study) of patients in which a surgical rehearsal was performed Surgical outcomes (ischemic time, positive surgicaly margins, hospitalization, post-operative complications, and estimated blood loss)

NA

2 surgeons completed the simulation and assessed the feasibility and accuracy of the model
as a simulation training plattrorm using subjective expert ratings as a simulation training plattorm using subjective expert ratings

Hydrogel (PVA) Validity study. The authors sough to demonstrate validity of the model after completion of the The
simulation by all participants Face validity: subjective ratings of model realism

Content validity: expert subjective ratings of model usefulness as an educational tool Construct validity: comparison of procedural metrics (ischemia time, blood loss,
positive margins and estimated blood loss) generated from each simulation between positive margins and estimated blood loss) generates
the three groups

Hydrogel (PVA) Validity study. The authors aimed to validate and incorporate CRPMS into a hydrogel model entrics of performance Study group consisted of $1^{\text {st }}$ year medical student and urology residents that reviewed $2 D$
CT scans followed by $3 \mathrm{D}$ modeles to accurately identify components of RENAL nephrometry
scores based on both modalities -specific 3D printed models in 5 patients scheduled for partial ephrectomy (4 robotic and 1 open) scores based on both modalities co
Clinical information provided 21 minutes, and negative margins were provided but without relevance to the benefit $3 \mathrm{D}$ models provided Change in surgical plan occurred in (30-50\%)

Most common changes were approach (trans or retroperitoneal approach) and hilar
clamping

Adequate fititing of the tumor specimens within the 3D mold was achieved in all 6 tumors $\$ 20.9-\$ 350.7$ Clinical data reported
All patients completed a successful off clamp partial nephrectomy
A single complication (urine leak) Maximal blood loss in a single case was reported (540 $\mathrm{mL}$ High ratings for surgical planning (from 7 to $9 / 10$ ) and anatomical accuracy (10/10) Athors reported accurate concordance between the $3 \mathrm{D}$ printed model and the histologic Approx. $\$ 500$ Ication of the index cancer lesion was noted, resulting in negative margins without

Surgeon reported satisfaction with the models that allowed tactile interaction with the Or wider excision of the NVBs around the palpable
tumors tumors
The authors report that models restored an aspect of tactile feedback lost in robotic
surgery Clinical data

No significant difference was seen between mean resection times in the model and patient (6:58 vs. 8:22 min, $\mathrm{P}=0.162)$ and tumor volumes between the excised model, Successful robotic partial nephrectomy was performed with a warm ischemia time $<30$ Comparison to case controls demonstrated a significantly lower blood loss (186 vs. 236
$\mathrm{~mL}, \mathrm{P}=0.01)$ Two vascular anastomoses between the hybrid donor renal vessels and the hybrid
ecipient's iliac vesselt in a mean anastomotic procedural time was 20 min per vessel.
Patency was also tested by intravascular injection of saline using a hypodermic needle demonstrating good anastomotic patency without leakage

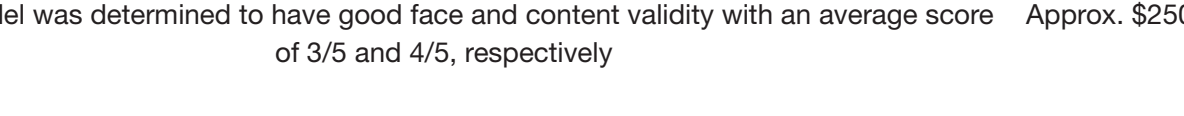

A significant difference was demonstrated in overall operative time $(P=0.003)$, ischemia
time $(P=0.04)$ positive margins $(P=0.002)$, and estimated good construct validity

Experts achieved superior margin status $(P=0.011)$. Nerve forces applied were significantly Approx. $\$ 250$ $(P=0.027)$ and total energy $(P=0.003)$. Higher force sensitivity $(S$ Subcategory of $G E A R S$ Score) and Total GEARS Score correlated with lower nerve forces applied with total energy $0.000)$, respectively, which was sing ceantly different between
D printed models lead to a more accurate characterization of 3 of the 4 components of
RENAL nephromety score

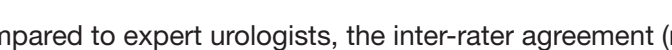

